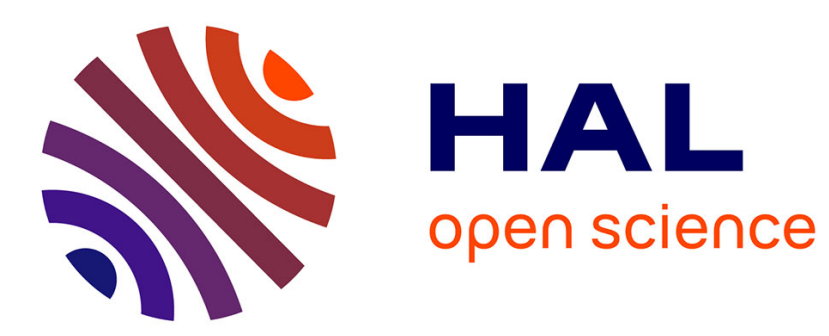

\title{
STRIVE: Socially-aware Three-tier Routing in Information-centric Vehicular Environment
}

\author{
Junaid Ahmed Khan, Yacine Ghamri-Doudane
}

\section{To cite this version:}

Junaid Ahmed Khan, Yacine Ghamri-Doudane. STRIVE: Socially-aware Three-tier Routing in Information-centric Vehicular Environment. IEEE Globecom 2016, Dec 2016, Washington D.C United States. hal-01361270

\author{
HAL Id: hal-01361270 \\ https://hal.science/hal-01361270
}

Submitted on 7 Sep 2016

HAL is a multi-disciplinary open access archive for the deposit and dissemination of scientific research documents, whether they are published or not. The documents may come from teaching and research institutions in France or abroad, or from public or private research centers.
L'archive ouverte pluridisciplinaire HAL, est destinée au dépôt et à la diffusion de documents scientifiques de niveau recherche, publiés ou non, émanant des établissements d'enseignement et de recherche français ou étrangers, des laboratoires publics ou privés. 


\title{
STRIVE: Socially-aware Three-tier Routing in Information-centric Vehicular Environment
}

\author{
Junaid Ahmed Khan*† and Yacine Ghamri-Doudane ${ }^{\dagger}$ \\ *University Paris-Est, LIGM Lab, Marne-la-Vallée, France \\ ${ }^{\dagger}$ L3i Lab, University of La Rochelle, France \\ junaid-ahmed.khan@univ-mlv.fr, yacine-ghamri@univ-lr.fr
}

\begin{abstract}
Content distribution in vehicular networks is greatly impaired by high mobility and intermittent connectivity. Socialaware content distribution schemes based on typical centrality metrics address the challenge, however they suffer due to their network-centric nature instead of information centric. We suggest to exploit the recently proposed information-centric networking architecture which cater the issue by decoupling host-user and support in-network caching at intermediate nodes. In this paper, we propose a novel information-centric social-aware content distribution protocol, STRIVE confining the broadcast nature of interest/content by routing it selectively towards high centrality information facilitator vehicles. We use a three-tier forwarding strategy to discover potential local and global information facilitators in an urban environment for efficient content delivery. The performance evaluation implements a scalable simulation environment deploying up to 2986 vehicles using realistic vehicular mobility traces. Simulation results show that our proposed novel vehicle centrality based content distribution protocol, STRIVE outperforms existing social content distribution metrics used in the literature.
\end{abstract}

Index Terms-Information-Centric Vehicular Networking, Social-aware Content Distribution, Vehicular Ad-hoc Networks.

\section{INTRODUCTION}

The technological improvements of modern vehicles and the increasing popularity of smart-phone applications emerge an increasing interest towards location-cognizant information. Interests for information regarding available parking lots, traffic/weather conditions, fuel prices, virtual tours to local attractions or snapshots of nearby resort areas are generated by applications targeting vehicles in a given area, regardless of their IP address. Moreover, video camera streams from vehicles facing an accident or a road emergency (flood, fire, etc.) can be considered in the local location-aware safety related content distribution category.

The difficulty to maintain end-to-end communication links in a vehicular network due to the intermittent connectivity and high mobility arise the need to design appropriate content distribution protocol for the latest application requirements. We observe a trend towards social-aware content distribution protocols where the best relay nodes are selected based on centrality measures [1], however it is not possible to compute typical centrality measures in highly unstable vehicular networks. Moreover, existing schemes follow a network-centric approach in order to analyze the network, thus, ignoring the content importance with respect to the user relevance and vehicular mobility.

To address this, we advocate to adapt Information-Centric Networking (ICN), a content-centric networking architecture proposed in [2] and [3] as the underlying routing protocol for vehicular networks. In ICN, a user broadcasts an interest for a content by its name, any corresponding host in the network replies back with the desired content. ICN aims to decouple the service from the host, thus removing content association to any physical location. It also offers in-network caching at intermediate nodes while forwarding and responding to subsequent user interests. However, broadcasting interests from a large number of nodes in ICN can result in a high network overhead. thus, the challenge is to minimize the interest broadcasts while achieving high user interest satisfaction with less network overhead.

In this paper, we propose the concept of socially aware information-centric vehicular networking where we present a novel routing protocol STRIVE. In STRIVE, the broadcast nature of interest forwarding in ICN is replaced by vehicle centrality based protocol, leveraging modern vehicles computing, caching and communicating capabilities. Thus, interests are forwarded only to vehicles with high centrality for efficient content distribution in a vehicular network. The next hop relay selection process is three-fold; first, we use our proposed local vehicle centrality scheme CarRank [4] to select the next-hop relay vehicle to forward a user interest at a local scope. In case the interest is not satisfied under a user specified deadline, the relay selection adapts our proposed global vehicle centrality metric GRank [5] to discover city-wide global information hubs to facilitate the desired content at the second-tier. In case of unavailability of the content at both, locally and globally central facilitator vehicles, the latest discovered facilitator finally requests the infrastructure to satisfy interest for a desired content.

The vehicle centrality algorithms CarRank and GRank enable each vehicle to autonomously find its importance in the network. Local importance measures a vehicle eligibility for short-term content distribution, while global importance enables the vehicle to facilitate long-term content availability in the network. To achieve this, we model the social content distribution protocol as a publish-subscribe ICN framework where vehicles subscribe to "pull" content of interest by selecting the best ranked "information facilitator vehicle" as the next hop relay nodes to publish content at the interested consumer. 
Information facilitator vehicles in the network are identified by their respective centrality score. The major contributions to this paper can be summarized as follows:

- A information-centric vehicular network as a publishsubscribe framework exploiting content-centric networking architecture where consumer "pulls" content of interest from the best provider in vehicular network.

- The first socially-aware content distribution protocol STRIVE proposed to forward interests/content to important information facilitator vehicles in the network.

- We validate the scalability as well as ICN compliance for STRIVE protocol by performing extensive simulations using around 2986 vehicles using realistic mobility traces in time evolving vehicular networks.

The rest of the paper is organized as follows. The next Section highlights the major related work. In Section III, we present the proposed the information-centric vehicular network model. The social content distribution protocol STRIVE is explained in Section IV. Section V describes the performance evaluation discussing the simulations. In Section VI we conclude the paper along some insight into our future directions.

\section{RELATED WORK}

Social-aware routing and content distribution has proved its performance in the past few years specially in Delay Tolerant Network (DTN) where it is difficult to maintain an end-toend communication link. Such protocols usually rely on well known network centrality schemes such as Degree, Closeness, Betweenness and Eigenvector centrality.By tweaking these centrality measures, algorithms such as BubbleRap [6] and MLSOR [7] are proposed, where nodes with high centrality score are preferred as next hop relay nodes for content dissemination and routing.

In a recent survey on Vehicular Social Networks (VSNs) [1], different sociability, security and applicability aspects of VSN paradigm are discussed. The authors advocate the emergence of social networks in vehicular scenarios due to increase in novel crowd-sourcing applications and increasing services based on social networking content sharing. In [8], the authors provide an extensive survey on different social-aware routing protocols for Delay Tolerant Networks (DTNs) and suggest to adapt the social metrics according to application requirements.

It is however unfeasible to use centrality-based popularity schemes to select the best ranked information facilitators in vehicular networks for multiple reasons; First, the rapid topological changes due to the high mobility of vehicles requires a continuous time varying analysis of the vehicular network which is unfeasible by a practical scheme. Indeed, typical schemes assume a static graph topology with respect to time where the temporal network characteristics of the network would be ignored. Second, centrality measures such as Betweenness, Closeness and Eigenvector centrality computation requires network wide parameters as a network-centric approach, instead of an information-centric approach. Third, existing schemes consider shortest path metric to compute a node's importance, while the highly dynamic vehicular network topologies does not ensure the existence of a stable path between nodes. Therefore, stable metrics adapted to vehicular networks need to be thought about for efficient content distribution.

\section{INFORMATION-CENTRIC VEHICULAR NETWORK}

The named-data networking concept introduced by the information-centric networking paradigm is capable to co-exist with the mobility and intermittent connectivity challenge in vehicular networks. The source-user decoupling allows the consumer interest for a desired content forwarded to "any" content provider independent of the underlying vehicular network connectivity. Therefore, we consider a vehicular network using a publish-subscribe ICN model for the efficient distribution of user relevant content.

\section{A. Publish-Subscribe ICN framework - The Big Picture}

The proposed publish-subscribe ICN paradigm allows the vehicle to subscribe for the following three roles:

1) Information Provider: An information provider vehicle acts as the content source to publish content. For example, it can subscribe itself to publish sensory information collected from urban streets using the vehicle embedded cameras and sensors.

2) Information Facilitator: Vehicle responsible to collect, relay and publish data generated by information provider vehicles as well as forwards the user interest for content to "facilitate" efficient content distribution.

3) Information Consumer: The vehicle subscribed to request different location-aware content from the information facilitators/providers with in the vehicular network are considered as information consumers to "pull" content in an informationcentric vehicular network.

Each ICN enabled vehicle maintains three routing parameters, (a) a Forwarding Information Base (FIB) as a routing table which maps content name components to interfaces. Each vehicle FIB is populated by the routes discovered using STRIVE. (b) a Pending Interest Table (PIT) to keep track of all the incoming interests that the vehicle has forwarded but not satisfied yet and (c) a Content Store (CS) as temporary cache to store content each intermediate vehicle has received while forwarding content. Since a named-data packet is meaningful independent of where it comes from or where it is forwarded, it can be cached to satisfy future interests.

We assume the city is divided into different urban zones using an ICN hierarchical naming convention which will be discussed in next section. A subscribed consumer vehicle forwards its interest to the an information facilitator vehicle in range which subsequently facilitates the content delivery with in the vehicular network. The Information facilitator vehicles look up their respective PIT for the desired content. Similarly a subscribed information provider vehicle can provide the content to information facilitators in order to publish content in the network.

The association between the information and the vehicles in an urban environment is achieved by clustering the city 
into different regions using ICN hierarchical naming convention "/region/road-section/information-type". Interests generated by consumers for content can specify the desired information type which comprises different Intelligent Transportation System (ITS) applications such as location-dependent safety warnings, road congestion information and location-independent infotainment applications.

\section{B. Network Model}

The vehicular network is modeled as a time varying undirected vehicular graph $G\left(\mathbb{V}(t), \mathbb{E}^{v}(t)\right)$, where $\mathbb{V}(t)=\{v\}$ is a set of vertices $v$, each representing a vehicle on the road at time $t . \mathbb{E}^{v}(t)=\left\{e_{j k}(t) \mid v_{j}, v_{k} \in \mathbb{V}, j \neq k\right\}$ is the set of edges $e_{j k}(t)$ modeling the existence of one hop communication link between vehicles $v_{j}$ and $v_{k}$ at time $t$. The number of edges $\mathbb{E}^{v}(t)$ depends on the transmission range of each vehicle. We assume it as a simple unit disk model bounded by its communication range. The city map is represented by the undirected graph $G\left(\mathbb{X}, \mathbb{E}^{x}\right)$, the set of vertices $\mathbb{X}=\{x\}$ contains different urban zones $x$ and the set of edges $\mathbb{E}^{x}=\left\{e_{p q} \mid x_{p}, x_{q} \in \mathbb{X}, p \neq q\right\}$ are their respective boundaries that connects different zones through the underlying road network. We also define the sub-graph $\Gamma_{v} \subset \mathbb{V}$ for a vehicle $v$ as the set of neighboring vehicles in its communication range at time instant $t$.

Information Association: Information association is defined as a bipartite graph $G(\mathbb{V}, \mathbb{X}, \mathbb{E})$, where $\mathbb{V}$ is the set the vertices in the vehicular graph $G\left(\mathbb{V}(t), \mathbb{E}^{v}(t)\right)$ and $\mathbb{X}$ is the set of locations in the city map $G\left(\mathbb{X}, \mathbb{E}^{x}\right)$. The edge $\mathbb{E}=$ $\left\{e_{i j} \mid v_{i} \in \mathbb{V}, x_{j} \in \mathbb{X}\right\}$ associates each vehicle to a set of regions $X_{v} \subset \mathbb{X}$ with respect to the user relevant content.

Definition 1: (Information Association Profile) The information association profile for each vehicle $v$ from the subgraph $X_{v} \subset \mathbb{X}$ is defined as the $N \times 1$ probability vector $P_{x}=\left[p_{x_{1}}, \ldots p_{x_{N}}\right]^{T}$, where $(\cdot)^{T}$ indicates the matrix transpose and $p_{x_{i}}$ is the probability of satisfying user interests for information associated to location $x_{i} \in X_{v}$, where $\sum_{x_{i} \in X_{v}} p_{x_{i}}=1$.

The regions are clustered using voronoi tessellation where the vehicles concentrated in an area are considered in a single voronoi region/zone $x \in G\left(\mathbb{X}, \mathbb{E}^{x}\right)$. The advantage of voronoi zones is that it retain the realism of city road structure, while covering the entire map. Additionally, it provides us an abstraction for the user relevant information regarding different neighborhoods, while not relying on individual road-sections.

For a temporal analysis of the vehicular network, we divide the time $T=\left(\overline{t_{1}}, \overline{t_{2}}, \ldots\right)$ into a sequence of regular timeslots, where the $k^{t h}$ time-slot is $\overline{t_{k}}=\left[t_{k}, t_{k+1}\right)$. We assume each vehicle knows the city map, i.e. the graph $G\left(\mathbb{X}, \mathbb{E}^{x}\right)$. We will refer to content/information or location/areas/zones interchangeably in the text since content are associated to different locations.

\section{STRIVE For CONTENT Distribution}

We propose a novel protocol for Socially-aware Routing in Information-centric Vehicular Environment (STRIVE) where consumers with common interests "pull" the content from important information facilitator vehicles in the network. The social awareness is considered by assuming vehicles encountering each other in a vehicular network constantly receives interests for content from neighboring vehicles regarding different location-cognizant information. Some of such information can be of more importance to the intended users in the network which the vehicle can easily recognize from the amount and frequency of user interests received for it. Therefore, a vehicle can consider an information as popular if it observes an increase in the number and frequency of user interests for the associated content in a respective time-slot. To identify the appropriate vehicles to be qualified as information facilitator vehicles in an urban environment is a challenging task. Therefore, in the following section, we discuss two novel vehicle centrality schemes to identify important information facilitator vehicles.

\section{A. Vehicle Centrality metric}

Each vehicle finds its centrality $C_{v}$ at the time instant $t_{k+1}$ from the known information in the current time-slot, where $t_{k}$ is the time instant at the beginning of the time-slot $\bar{t}_{k}$.

1) Local Centrality - CarRank: It is difficult to use the vehicle contact frequency and duration to decide its importance in the network due to the rapid changes in the time evolving vehicular network topology. To overcome this, we use CarRank which simultaneously considers three novel albeit essential parameters, the information importance, the vehicle spatiotemporal availability and its network connectivity. The user's interest satisfaction frequency for a content is also considered as a key metric for a vehicle's importance as it regularly responds to user interests. The interests are assumed to be generated and received from the neighboring vehicles using multi-hop interest forwarding. We consider the following local parameters known to the vehicle for analytically finding its importance:

- Information importance: Information importance measures vehicle relevance to users for a particular content, i.e. The interest-response frequency is a vital factor to classify a content's importance.

- Spatio-temporal availability: It reflects the social-behavior based on the vehicle's habitual routes as a factor of the daily commute. Spatial availability reflects the vehicle's recursive presence in an area, while temporal availability refers to its relevance in time for a location.

- Neighborhood importance: Neighborhood importance shows vehicle topological connectivity in order to be capable to distribute information. An easily reachable and well connected vehicle in a network topology can act as an efficient facilitator.

The vehicle first classify the information associated to it taking into consideration the relevance to the users interest. It then considers the associated information popularity to find its relative importance in the network using CarRank algorithm. CarRank determines a vehicle eligibility as a local information 
facilitator responsible for the efficient content distribution:

$$
\begin{array}{r}
L C_{v}\left(t_{k+1}\right)=\theta \times L C_{v}\left(t_{k}\right)+(1-\theta) \times\left[\alpha f_{I}^{v}\left(t_{k+1}\right)\right. \\
\left.+\beta f_{T, X}^{v}\left(t_{k+1}\right)+\gamma f_{\Gamma}^{v}\left(t_{k+1}\right)\right]
\end{array}
$$

where $f_{I}^{v}, f_{T, X}^{v}$ and $f_{\Gamma}^{v}$ are the importance functions for the information, vehicle spatio-temporal availability and vehicle neighborhood respectively. Each function's contribution is normalized by the terms $\alpha, \beta$ and $\gamma$, where $\alpha+\beta+\gamma=1$, where $\theta \in[0,1]$ allows the vehicle to increase its importance with respect to the previous time-slot. The impact of each parameter differs with respect to different applications. For example, if the vehicle is located in a better connected neighborhood, it can easily spread information. Therefore, the corresponding vehicle weights the information importance along the neighborhood more than the spatio-temporal availability function.

2) Global Centrality - GRank: Inspired from the concept of communicability in complex networks [9], GRank, a global vehicle centrality scheme allows a vehicle to use a new stable metric named "Information communicability" to rank different locations in the city and rank itself accordingly. Using GRank, the vehicle finds each location reachability and popularity taking into consideration the user interest satisfaction related to the location. It also considers its mobility pattern between different locations in the city along its availability in each location. Vehicles available in popular locations in the city qualify as important information facilitator vehicles with higher vehicle centrality score in the network.

The importance of a location $x_{i}$ with respect to the vehicle availability pattern as well as the associated information reachability is given as $\rho_{x_{i}}^{v}\left(t_{k+1}\right)=\pi_{x_{i}} \cdot f_{x_{i}}^{v}\left(t_{k+1}\right)$, where $\pi_{x_{i}}$ yields the probability of the vehicle availability in the region and $f_{x_{i}}^{v}$ considers the respective information centrality function. The information global centrality for each location can now be defined as:

$$
G_{x_{i}}^{v}\left(t_{k+1}\right)=\delta \times \rho_{x_{i}}^{v}\left(t_{k+1}\right)+(1-\delta) \times \rho_{x_{i}}^{v}\left(t_{k}\right)
$$

where $\rho_{x_{i}}^{v}\left(t_{k}\right)$ is maintained by each vehicle as the location importance value at the time $t_{k}$. The parameter $\delta \in[0,1]$ is used to tune between the recent location popularity and overall location popularity. Thus, we can identify popular locations at time instant $t_{k+1}$ in the city with the maximum global centrality $\arg \max G_{x_{i}}^{v}\left(t_{k+1}\right)$ with respect to all facilitator vehicles. $v \in \mathbb{V}, x_{i} \in \mathbb{X}$ However, popularity of locations depends on several factors such as the information-type depending on the application requirements as well as time of the day. Similarly, we can use the maximum location importance $\max _{v \in \mathbb{V}, x \in \mathbb{X}} \rho_{x_{i}}^{v}$ to identify popular neighborhoods for a longer time span.

Each vehicle information association profile plays an important role in deciding its importance in the network. Thus, it should receive and subsequently satisfy more frequently the interests for information regarding the popular regions in the city. The vehicle centrality function at the time instant $t_{k+1}$ is given as the average information global centrality for all associated locations:

$$
f_{G}^{v}\left(t_{k+1}\right)=\frac{1}{\left|X_{v}\right|} \sum_{x_{i} \in X_{v}} G_{x_{i}}^{v}\left(t_{k+1}\right),
$$

For a vehicle $v$, the vehicle global centrality $G C_{v}\left(t_{k+1}\right)$ for the next time instant $t_{k+1}$ is updated as the Exponential Weighted Moving Average (EWMA) function of the current and previous global centrality as shown in the relation below:

$$
G C_{v}\left(t_{k+1}\right)=\theta \times G C_{v}\left(t_{k}\right)+(1-\theta) \times f_{G}^{v}\left(t_{k+1}\right),
$$

where $\theta \in[0,1]$ is the tuning parameter which allows the vehicle to adjust its importance with respect to the previous time-slot, $G C_{v}\left(t_{k}\right)$ is the vehicle global centrality at the beginning of the current time-slot and $f_{G}^{v}\left(t_{k+1}\right)$ is the vehicle global centrality computed at the end of the current time-slot.

The rationale behind considering both CarRank and GRank can be explained by the fact that each interest specifies two satisfaction deadlines $I_{\max }$ and $I_{\min }$, where $I_{\max } \geq I_{\min }$ indicates the maximum and minimum threshold time to provide the corresponding content. Thus in case the interest cannot be satisfied by a local facilitator vehicle in the vicinity by an initial threshold $I_{\min }$, the interests can be forwarded to more globally central vehicles till $I_{\max }$; the maximum interest deadline to avoid bandwidth and time utilization.

Definition 2: (Facilitator Centrality) The facilitator centrality is defined as $\tilde{\mathrm{C}}_{v}\left(t_{k+1}\right)=C_{v}\left(t_{k+1}\right) \times P_{x}$, where $C_{v}$ is the vehicle centrality which can be either $L C_{v}$, the local centrality CarRank or $G C_{v}$, the global centrality GRank depending on the interest deadline $I_{d}$, the probability vector $P_{x}$ is the respective information association profile. For efficient content distribution, the vehicle association with respect to the current location is extracted from the information association profile $P_{x}$ where $p_{x_{i}} \neq 0$, for $x_{i} \in X_{v}$ at the current location.

The information association profile measures the vehicles reliability as an information facilitator at the current location. Thus, the facilitator centrality takes into account both the vehicle centrality as well as its association to the current location in order to be considered as information facilitator vehicle.

\section{B. Content Distribution Protocol}

The centrality-based content distribution protocol leverage the facilitator centrality to forward consumer interests for content as well as route the content from the corresponding providers. The information provider as well as the consumer search for a near-by information facilitator vehicle using its centrality score to forward interest/content. Our proposed content distribution protocol provides a hybrid solution using the ICN inherent pull based content retrieval for the consumer and a push based approach for the provider to publish content. The information facilitator discovery is described as following:

Information Facilitator Discovery: The facilitator discovery process allows a vehicle $u \in \mathbb{V}$ to search in its vicinity the highest centrality facilitator vehicle $u^{*}=\underset{v \in \Gamma_{u}}{\arg \max }\left\{\tilde{\mathrm{C}}_{v}\right\}$ using the $\operatorname{FACILITATOR}(u)$ function. It compares the facilitator 


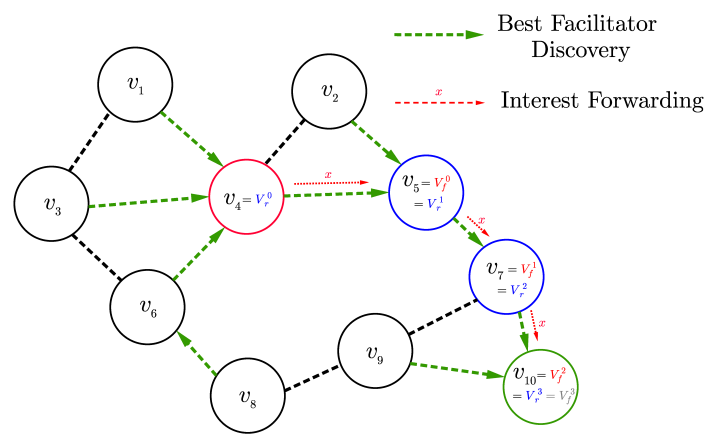

Figure 1: Facilitator Discovery Process

centrality score of all the neighboring vehicles and returns the best facilitator centrality vehicle among the vehicles in range for a vehicle $u$, where $\Gamma_{u}$ is the set of neighboring vehicles.

The $\operatorname{PROVIDER}(x, u)$ function assigns a vehicle as provider vehicle to publish the content $x$ for the consumer vehicle $u$. The publishing of content can be either solicited or non solicited. In the case of solicited interest, the provider can publish content $x$ destined for $u$ with a near-by information facilitator $u^{*}$ using PUBLISH $\left(x, u^{*}, u\right)$ function. Similarly, a non-solicited publish with a near-by facilitator can be performed by an information facilitator discovery process initiated anytime by the information provider. The CONTENT $\left(x, v_{f}^{m}\right)$ function is used for the content availability check at the Content Store (CS) of each intermediate vehicle $v_{r}^{m}$.

We adapt three-tier next hop relay selection scheme where the initially facilitator discovery uses CarRank to forward the interest till the first interest deadline threshold $I_{\min }$ is reached. The process then replaces the local centrality metric CarRank by GRank, the global centrality metric in the second phase for efficient content retrieval in the network, and finally the internet is requested in case of unavailability of content in the network. The three-tier facilitator discovery address the following objective; first it minimize the broadcast nature to few selective number of vehicles which are capable to facilitate user interest in a local vicinity using a local centrality scheme CarRank. Second, the interest deadlines invokes the need to enhance the search on a global city-wide scale by finding globally important next hop relay vehicles, thus minimizing interest overhead by targeting set of selected global information hubs. Algorithm 1 describes the social content distribution protocol. Consumer vehicle $v \in \mathbb{V}$ generates interests for content $x \in \mathbb{X}$ as $\operatorname{INTEREST}(x, v)$ towards to the best ranked facilitator in the vicinity. Lines 3-5 initializes the facilitator discovery process where $v_{f}^{o}$ is assigned as the initial facilitator for the consumer $v$ in its vicinity. The initial facilitator vehicle $v_{r}^{o}$ is assigned to requester $v$ considering it responsible for itself.

The facilitator discovery process in Lines 6-10 continues to search for the content at each intermediate relay vehicle by constantly discovering the next best ranked vehicle $v_{r}^{m+1}$ in the vicinity of each intermediate relay vehicle $v_{f}^{m}$. Thus,

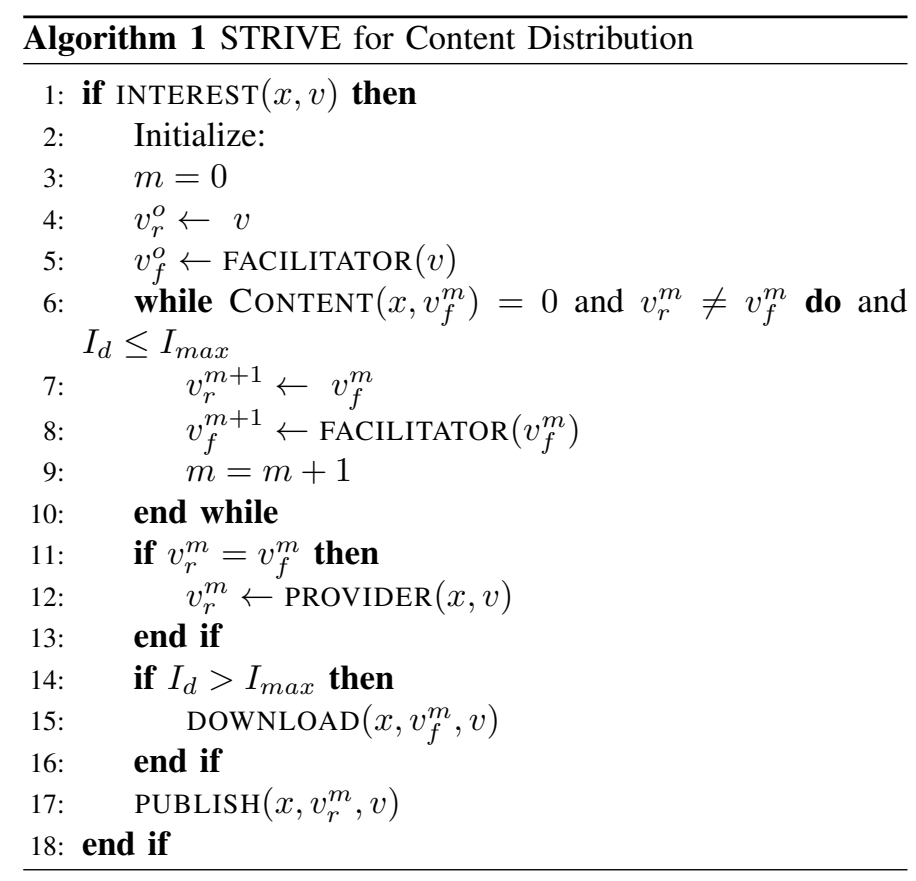

each relay vehicle becomes the responsible vehicle to facilitate the content. If it is unable to find the content in its CS, it performs a facilitator discovery to find a vehicle with higher vehicle centrality score and a Pending Interest Table (PIT) entry is created. The centrality metric switch to the GRank upon the expiry of the initial interest deadline $I_{\min }$. The process is repeated at each intermediate facilitator till either the desired content is found or there are no more facilitators to discover as shown in Figure 1.

\section{Convergence and Failure Recovery}

The convergence of the facilitator discovery process of STRIVE is two-fold, the first obvious convergence occurs when the desired content is available at the corresponding facilitator. In this case, the content is published at the consumer vehicle (Line 17) following a reverse path to the initial requester using breadcrumbs left in the PIT at each intermediate node. The intermediate vehicles subsequently populates the corresponding Forwarding Information Base (FIB) entry for the content $x$. In case the content is not available and there are no further facilitators to discover as mentioned in Lines 11-14, the responsible vehicle $v_{r}^{m}$ declares itself as the content provider to publish content at the consumer vehicle.

STRIVE integrates a failure recovery system which is achieved by the latest global information facilitator vehicles. For an interest generated by a consumer vehicle, in case the latest discovered global information facilitator vehicle $v_{f}^{m}$ is unable to satisfy the interest upon the expiry of the maximum threshold $I_{\max }$ for the interest deadline in Lines 1416. It finally requests the infrastructure network as the last resort to provide the desired content. This is achieved by the $\operatorname{DOWNLOAD}\left(x, v_{f}^{m}, v\right)$ function, where the last discovered facilitator $v_{f}^{m}$ downloads the content $x$ for the consumer 
Table I: Simulation Parameters

\begin{tabular}{l|l}
\hline Parameter & Value \\
\hline Simulation platform & NS-3 \\
Number of nodes & 2986 \\
Mobility trace & Cologne, Germany \\
Area & $6 X 6 \mathrm{~km}^{2}$ (city center) \\
Duration & 1 hour \\
Communication range & $100 \mathrm{~m}$ \\
Packet size & 1024 bytes \\
Time granularity & $1 \mathrm{sec}$ \\
Simulation Runs & 10 \\
\hline
\end{tabular}

vehicle $v$. Thus, we reduce the dependency on the infrastructure network by initially discovering content at local important vehicles closer to the end user, then in case of failure, download the desired content from the infrastructure for the efficient satisfaction of the respective consumer interest.

\section{Performance Evaluation}

The basic requirement for evaluating the efficiency of STRIVE is its scalability. Therefore, we use ndnSIM module [10] for Network Simulator-3 (NS-3) as a scalable simulation platform for 2986 vehicles under a realistic mobility scenario using traces from Cologne, Germany. To the best of our knowledge, it is considered as the most accurate mobility trace available for Vehicular Networks [11]. The vehicle availability as well as its mobility pattern is extracted using this trace. The simulation parameters are summarized in Table I, followed by a description of the simulation scenario implemented for the performance evaluation.

\section{A. Simulation Scenario}

We simulate a urban vehicular network implementing the Named Data Networking (NDN) communication model where the named-data networking architecture replaces the traditional IPv4/v6 NS-3 network-layer module. The simulation scenario implements the following applications:

1) Consumer: Consumer vehicles are the potential users planning to visit an area. Each consumer vehicle generates an interest for a content associated to a location in the city, which is routed to provider vehicles.

2) Provider: We define a vehicle to be the content provider in the network for the areas visited in a time-slot before the consumer interest generation time. The areas visited are considered as locations associated with the provider.

3) Facilitator: Vehicles satisfied incoming requests generated form consumers regularly computes their centrality score to consider themselves as information facilitators. Similarly, constant content forwarding and cache hits also counts towards the facilitator centrality score.

We assume the interests follow a Zipf distribution. Interests generated for content associated to different locations are forwarded to the discovered information facilitators which are responsible to facilitate the desired content by finding for the information provider. Any provider acting as source for an area upon receiving the interest publishes the desired content.

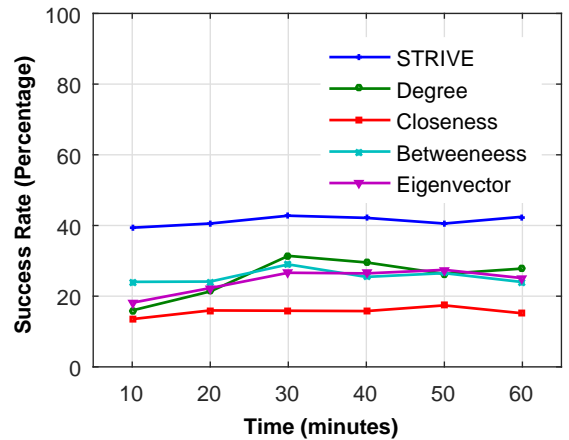

Figure 2: Success rate for the generated interests satisfied over time using different social metrics

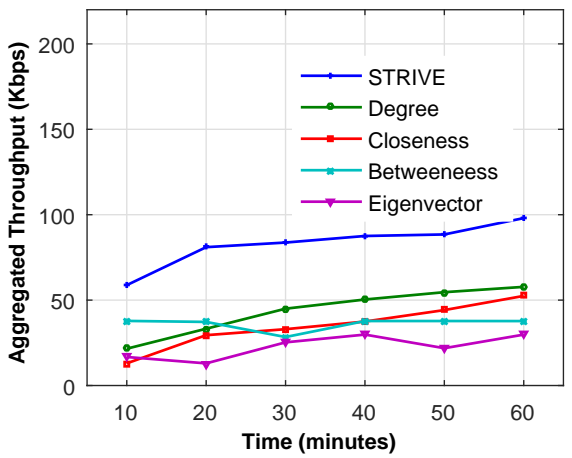

Figure 3: Aggregated throughput comparison over time at information facilitators selected using different social metrics

\section{B. Simulation Results}

For better performance analysis of STRIVE in different simulation scenarios, we compare it with the centrality metrics used by most of state of the art social-aware routing schemes. The following performance metrics are considered for an average of ten simulation runs, (a) success rate for satisfying consumer interests in the network, (b) aggregated per node throughput at the information facilitators, (c) aggregated content store (cache) hit rate at the information facilitators:

1) Success Rate: Success rate refers to the percentage of the generated consumer interests successfully satisfied compared to the generated interests in the simulation duration taking into account the interests overhead in the network. STRIVE is compared with the state of the art centrality schemes as benchmark. Figure 2 shows the percentage of consumer interests for different locations successfully satisfied by the corresponding information facilitators/providers. We observe that forwarding the generated consumer interest towards a socially important vehicle using CarRank and GRank as a metric results in more number successful interest satisfaction. The vehicles identified by STRIVE satisfied around $40 \%$ of the generated interests compared to other centrality metrics despite high mobility and intermittent connectivity. It is because typical centrality schemes only takes into account physical topology towards computing a node importance in the network, ignoring the satisfied user interests. Thus a high success rate by STRIVE 


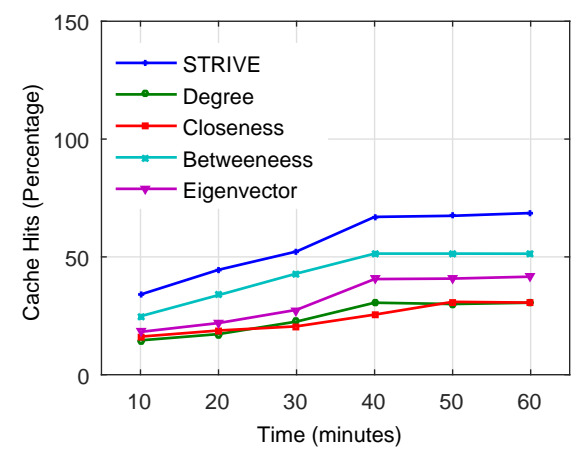

Figure 4: Cache hits comparison over time at the information facilitators selected using different social metrics

indicates a substantial reduction in interests flooding.

2) Aggregated Throughput: We also evaluate STRIVE by analyzing the aggregated throughput at the selected facilitator vehicles in the network. The facilitator vehicles are identified by their vehicle centrality score as well as applying the existing centrality schemes computed over the entire simulation duration averaged over ten set of simulations. Figure 3 shows the aggregated per node throughput at the facilitator nodes identified by using each centrality scheme. The vehicles identified as facilitators by STRIVE yields twice more throughput compared to those identified by other schemes. Temporal analysis of vehicular network reveals an increase in the facilitators throughput over time. The reason is the increasing participation of best ranked facilitators towards content distribution in the network. Similar increase in facilitator participation is also seen for degree and closeness centrality. However, betweenness and closeness centrality do not show such increase. The reason our proposed vehicle centrality outperformed all schemes as it incorporates additional factors towards vehicle importance computation such as the information importance and the spatiotemporal availability, while other schemes rely only on topological measures (node degree or shortest paths) towards facilitator selection for efficient content distribution.

3) ICN Evaluation - In-Network Caching: We evaluate the ICN built-in feature of In-Network caching at intermediate nodes at the selected facilitator vehicles. For this purpose, we compute the cache hit rate at the facilitator vehicles. A second successful response by a vehicle for the same content is considered a cache hit. The cumulative cache hit rate is computed for the entire simulation duration averaged over ten set of simulations. Figure 4 shows the cache hit rate for the facilitator vehicles identified by each centrality scheme. The vehicles identified by STRIVE yield a higher hit rate than all the other schemes. This is because we consider content popularity as a key factor, thus, the vehicle containing important information responds and subsequently cache more frequently compared to other vehicles.

We also observe that the vehicles identified using betweeness centrality follows STRIVE yielding better cache hit rate due their frequent availability as intermediate bridges at most of the shortest paths, thus allowing them to cache more content. Moreover, the intermediate facilitators identified by our vehicle centrality scheme cached more important content due to their better neighborhood connectivity and spatio-temporal availability in the network. This proves that in-network caching offered by ICN along the proposed vehicle centrality scheme overcomes the mobility and intermittent connectivity constraints in vehicular network for efficient content distribution.

\section{Conclusions And Future Directions}

This paper proposed STRIVE, a social-aware informationcentric routing protocol for vehicular networks. We used our proposed vehicle centrality metrics CarRank and GRank to identify important vehicles. Consumer interests for desired content are routed to the information facilitator vehicles with the best vehicle centrality score. Such vehicles are responsible for efficient content distribution in order to facilitate consumer interests in an publish-subscribe information-centric vehicular network. Comparative analysis with state of the art social routing metrics by performing extensive simulations using realistic mobility traces showed that STRIVE outperforms the existing socially-aware schemes.

Future work address the in-network caching support provided by ICN in order to cache at the best ranked information facilitator vehicles for efficient content delivery. Such information facilitators are selected for different smart city applications minimizing network load and cost by placing content closer to the citizens.

\section{REFERENCES}

[1] A. M. Vegni and V. Loscri, "A survey on vehicular social networks," Communications Surveys \& Tutorials, IEEE, vol. 17, no. 4, pp. 23972419, 2015.

[2] M. Amadeo, C. Campolo, A. Molinaro, and G. Ruggeri, "Content-centric wireless networking: A survey," Computer Networks, vol. 72, pp. 1-13, 2014.

[3] M. Gerla, E.-K. Lee, G. Pau, and U. Lee, "Internet of vehicles: From intelligent grid to autonomous cars and vehicular clouds," in Internet of Things (WF-IoT), World Forum on. IEEE, 2014, pp. 241-246.

[4] J. A. Khan and Y. Ghamri-Doudane, "Carrank: An information-centric identification of important smart vehicles for urban sensing," in 14th International Symposium on Network Computing and Applications, NCA 2015 IEEE.

[5] J. A. Khan, Y. Ghamri-Doudane, and D. Botvich, "Grank - an information-centric autonomous and distributed ranking of popular smart vehicles," in Globecom 2015 Proceedings IEEE.

[6] P. Hui, J. Crowcroft, and E. Yoneki, "Bubble rap: Social-based forwarding in delay-tolerant networks," Mobile Computing, IEEE Transactions on, vol. 10, no. 11, pp. 1576-1589, 2011.

[7] A. Socievole, E. Yoneki, F. De Rango, and J. Crowcroft, "Ml-sor: Message routing using multi-layer social networks in opportunistic communications," Computer Networks, vol. 81, pp. 201-219, 2015.

[8] K. Wei, X. Liang, and K. Xu, "A survey of social-aware routing protocols in delay tolerant networks: Applications, taxonomy and design-related issues," Communications Surveys \& Tutorials, IEEE, vol. 16, no. 1, pp. 556-578, 2014.

[9] E. Estrada and N. Hatano, "Communicability in complex networks," Physical Review E, vol. 77, no. 3, p. 036111, 2008.

[10] A. Afanasyev, I. Moiseenko, and L. Zhang, "ndnSIM: NDN simulator for NS-3," NDN, Technical Report NDN-0005, October 2012. [Online]. Available: http://named-data.net/techreports.html

[11] S. Uppoor, O. Trullols-Cruces, M. Fiore, and J. M. Barcelo-Ordinas, "Generation and analysis of a large-scale urban vehicular mobility dataset," Mobile Computing, IEEE Transactions on, vol. 13, no. 5, pp. 1061-1075, 2014. 